\title{
Soliton-Like Spherical Symmetric Solutions to the Electromagnetic and Scalar Nonlinear Induction Field Equations in the General Relativity Theory
}

\author{
Arnaud E. Yamadjako1,2* ${ }^{\mathbb{0}}$, Alain Adomou, ${ }^{1,3}$, Yélomè J. F. Kpomahou², Jonas Edou', Siaka Massou ${ }^{1}$ \\ ${ }^{1}$ Department of Physics, University of Abomey-Calavi, Abomey-Calavi, Benin \\ ${ }^{2}$ Department of Industrial and Technical Sciences, ENSET-Lokossa, UNSTIM, Abomey, Benin \\ ${ }^{3}$ National Higher Institute of Industrial Technology, National University of Sciences, Technologies, Engineering and Mathematics \\ of Abomey, UNSTIM, Abomey, Benin \\ Email: *arnouard@gmail.com
}

How to cite this paper: Yamadjako, A.E., Adomou, A., Kpomahou, Y.J.F., Edou, J. and Massou, S. (2022) Soliton-Like Spherical Symmetric Solutions to the Electromagnetic and Scalar Nonlinear Induction Field Equations in the General Relativity Theory. Journal of High Energy Physics, Gravitation and Cosmology, 8, 147-163.

https://doi.org/10.4236/jhepgc.2022.81011

Received: November 11, 2021

Accepted: January 3, 2022

Published: January 6, 2022

Copyright $\odot 2022$ by author(s) and Scientific Research Publishing Inc. This work is licensed under the Creative Commons Attribution International License (CC BY 4.0).

http://creativecommons.org/licenses/by/4.0/

\begin{abstract}
In this paper, we have used the static spherical symmetric metric. The parameter of the nonlinearity fields is included in the arbitrary function characterizing the interaction between the electromagnetic and scalar fields. Taking into account the own gravitational field of elementary particles, we have obtained exact static spherical symmetric solutions to the electromagnetic and scalar field equations of nonlinear induction. Considering all forms of the solution of Liouville equation, we proved that the metric functions are regular with localized energy density. Moreover, the total energy of the nonlinear induction fields is bounded and the total charge of the elementary particles has a finite value (soliton-like). In the flat space-time, soliton-like solutions exist.
\end{abstract}

\section{Keywords}

Interaction, Scalar, Electromagnetic, Gravitational Fields, Description, Configuration, Elementary Particles

\section{Introduction}

In classical physics, elementary particles are considered as material points. Moreover, in the Standard Model (SM), the Grand Unification Theories (GUT), the Super Symmetry Super Strings (SUSY), the theory of gravitation is absent [1]. From experimental results and in [2], elementary particles are extended objects with a complex spatial configuration. The theories or models (SM, GUT, SUSY) 
do not therefore solve the problem of the description of the configuration of elementary particles. This description, with all their physical characteristics is only possible in the framework of field interaction theory and in quantum mechanics [3]. In particle physics, soliton-like solutions to nonlinear differential field equations are used as models to describe the complex spatial configuration of elementary particles [4] [5].

The soliton is a particular solution of nonlinear differential equations, spatially localized and of exceptional stability [6] [7]. The notion of soliton has given the opening to a series of scientific works. For example, the basis of soliton was elaborated in [8] [9]. The resolution of the KdV equation in [10] leads to the theoretical interpretation of J. S. Russell observation [11]. The work of [12] [13] [14] and the Carter-Penrose diagram, are references for studying the singularity of static spherical and symmetric solutions. In [15], the authors generalized the solutions to the Born-Infeld nonlinear electrodynamics equation based on the results of [16]. In [17], the resolution to the equation of scalar, electromagnetic and gravitational fields leads to the obtaining of static spherical symmetry solutions. The authors affirmed the existence of a system of particle-antiparticle pairs. In [18], exact Kink-Like static plane symmetric solutions to the self-consistent system of electromagnetic, scalar and gravitational field equations are obtained. It was shown that under certain choice of the interaction Lagrangian, the solutions are regular and have localized energy (like-soliton). In [19], the authors determined, soliton-like solutions to the nonlinear electromagnetic field equation interacting with the scalar field in the spherical and/or cylindrical symmetric metric in the presence of the own gravitational field of elementary particle by studying only the special case where $S(k, \xi)=\xi$. The authors found that with the calibrated invariance function $P(I)=P_{0}(\lambda I-N)^{2}$ and under the condition $\lambda I>N$, the component of metric tensor $g_{00}$ is regular and the total energy of the nonlinear induction fields is finite in the configuration where the mass and charge are zero. The solution obtained describes a massive system $\left(A(0)=\frac{m}{q}\right)$ but the total charge of elementary particles $Q$ has not been examined. In [20] [21] [22] [23] static spherical symmetric soliton-like solutions to the spinoriel and gravitational field equations were established using several bilinear invariants.

The objective of this research work is to determine the exact static spherical symmetric soliton-like solutions to the electromagnetic and scalar nonlinear induction field equations taking into account the own gravitational field of elementary particles, describing a massless system, considering all forms of the function $S(k, \xi)$, using the calibrated invariance function $P(I)=P_{0}(N-\lambda I)^{2}$, under the condition $\lambda I<N$. To achieve this, Section 2 of this work gives a brief overview of the basic equations. In Section 3, the established solutions are presented. A discussion and a comparative study are made in Section 4. The role of the own gravitational field of elementary particles is studied in Section 5. Sec- 
tion 6 is dedicated to the conclusion and future work.

\section{Basic Equations}

In general relativity, Einstein's equation is:

$$
G_{\mu}^{v}=-\chi T_{\mu}^{v},
$$

where $\left(G_{\mu}^{v}\right)$ is the Einstein tensor, $(\chi)$ is the Einstein gravitational constant and $\left(T_{\mu}^{v}\right)$ is the energy-momentum metric tensor. The static spherical symmetric metric is defined by:

$$
\mathrm{d} s^{2}=\mathrm{e}^{2 \gamma} \mathrm{d} t^{2}-\mathrm{e}^{2 \alpha} \mathrm{d} \xi^{2}-\mathrm{e}^{2 \beta}\left[\mathrm{d} \theta^{2}+\sin ^{2}(\theta) \mathrm{d} \varphi^{2}\right],
$$

where the functions $\alpha, \beta$ and $\gamma$ depend only on the radial component $\xi=\frac{1}{r}$ and obey the coordinate relation [24]:

$$
\alpha=2 \beta+\gamma \text {. }
$$

From (1), (2) and (3), the non-zero components of Einstein's tensor equation are [24]:

$$
\begin{gathered}
G_{0}^{0}=\mathrm{e}^{-2 \alpha}\left(2 \beta^{\prime \prime}-2 \gamma^{\prime} \beta^{\prime}-\beta^{\prime 2}\right)-\mathrm{e}^{-2 \beta}=-\chi T_{0}^{0} \\
G_{1}^{1}=\mathrm{e}^{-2 \alpha}\left(2 \gamma^{\prime} \beta^{\prime}+\beta^{\prime 2}\right)-\mathrm{e}^{-2 \beta}=-\chi T_{1}^{1} \\
G_{2}^{2}=\mathrm{e}^{-2 \alpha}\left(\beta^{\prime \prime}+\gamma^{\prime \prime}-2 \gamma^{\prime} \beta^{\prime}-\beta^{\prime 2}\right)=-\chi T_{2}^{2} \\
G_{2}^{2}=G_{3}^{3} \\
T_{2}^{2}=T_{3}^{3}
\end{gathered}
$$

where (') denotes the first derivative with respect to $\xi$.

The Lagrangian of the electromagnetic and scalar nonlinear fields in interaction, taking into account the own gravitational field of the elementary particles has considered in the form:

$$
L=\frac{R}{2 \chi}-\frac{1}{4} F_{i j} F^{i j}+\frac{1}{2} \varphi_{, i} \varphi^{, i} \psi(I),
$$

where $I=A_{i} A^{i}$ is the chronometric invariant; $A_{i}(A(\xi), 0,0,0)$ is the 4 -vector potential; $\psi(I)=1+\lambda \phi(I)$ is an arbitrary function characterizing the interaction between the electromagnetic and scalar nonlinear fields, $\lambda$ represents the parameter of the nonlinearity

In the absence of a current source, the scalar and electromagnetic field equations corresponding to the Lagrangian (9) take the form [19]:

$$
\begin{gathered}
\frac{1}{\sqrt{-g}} \frac{\partial}{\partial \xi^{v}}\left[\sqrt{-g} g^{\nu \mu} \varphi_{, \mu} \psi(I)\right]=0, \\
\frac{1}{\sqrt{-g}} \frac{\partial}{\partial \xi^{\mu}}\left[\sqrt{-g} F^{v \mu}\right]-\varphi_{, i} \varphi^{, i} \psi_{I}(I) A^{v}=0 .
\end{gathered}
$$

The energy-momentum metric tensor is defined by:

$$
\begin{aligned}
T_{\mu}^{v}= & \varphi_{, \mu} \varphi^{, v} \psi(I)-F_{\mu i} F^{v i}+\varphi_{, i} \varphi^{, i} \psi_{I}(I) A^{v} A_{\mu} \\
& -\delta_{\mu}^{v}\left[-\frac{1}{4} F_{i j} F^{i j}+\frac{1}{2}\left(\varphi_{, i} \varphi^{, i}\right) \psi(I)\right] .
\end{aligned}
$$


From (12), its non-zero components are:

$$
\begin{gathered}
T_{0}^{0}=\frac{1}{2} \mathrm{e}^{-2 \alpha}\left[\mathrm{e}^{-2 \gamma}\left(A^{\prime}\right)^{2}+C^{2} P(I)+2 \mathrm{e}^{-2 \gamma} C^{2} P_{I}(I)(A)^{2}\right] \\
T_{1}^{1}=\frac{1}{2} \mathrm{e}^{-2 \alpha}\left[-C^{2} P(I)+\mathrm{e}^{-2 \gamma}\left(A^{\prime}\right)^{2}\right] ; \\
T_{1}^{1}=-T_{2}^{2}=-T_{3}^{3} .
\end{gathered}
$$

The relation (10) has a solution:

$$
\frac{\mathrm{d} \varphi}{\mathrm{d} \xi}=\frac{C}{\psi(I)}=C P(I) .
$$

The electromagnetic field (11) becomes:

$$
\left(\mathrm{e}^{-2 \gamma} A^{\prime}\right)^{\prime}-C^{2} \mathrm{e}^{-2 \gamma} P_{I}(I) A=0 .
$$

The sum of (5) and (6), reduce to the Liouville equation [24]:

$$
(\beta+\gamma)^{\prime \prime}=\mathrm{e}^{2(\beta+\gamma)},
$$

which is integrated giving:

$$
\mathrm{e}^{-(\beta+\gamma)}=S(k, \xi)= \begin{cases}\frac{\sinh k \xi}{k}, & k>0 \\ \xi, & k=0 \\ \frac{\sin k \xi}{k}, & k<0\end{cases}
$$

The sum of (4) and (5), leads to:

$$
\beta^{\prime \prime}-\mathrm{e}^{2(\beta+\gamma)}=-\frac{\chi}{2} \mathrm{e}^{-2 \gamma}\left[-C^{2} P(I)+\mathrm{e}^{-2 \gamma}\left(A^{\prime}\right)^{2}\right] .
$$

From (17), (18) and (20), we draw:

$$
\gamma^{\prime \prime}=\frac{1}{2} \chi\left(A A^{\prime} \mathrm{e}^{-2 \gamma}\right)^{\prime} .
$$

The first integration of (21) gives:

$$
\gamma^{\prime}(\xi)=\frac{1}{2} \chi\left(A A^{\prime} \mathrm{e}^{-2 \gamma}\right)+Y, \quad Y=\text { const. }
$$

For $Y=0$, the solution of (22) is:

$$
\mathrm{e}^{2 \gamma}=\frac{\chi A^{2}}{2}+H, \quad H=\text { const. }
$$

The relation (23) introduced in (17) gives:

$$
\begin{aligned}
& \pm\left(\xi+\xi_{0}\right)=\int \frac{\mathrm{d} A}{\left(\frac{\chi A^{2}}{2}+H\right) \sqrt{C^{2} P(I)+K}}, \\
& \xi_{0}=\text { const, } \quad K=\text { const. }
\end{aligned}
$$

From the regularity conditions established in [19] and [25], we fix $H=1$, 
$K=0$ and assume $\xi_{0}=0$. Thus, the relations (23) and (24) become:

$$
\mathrm{e}^{2 \gamma}=\frac{\chi A^{2}}{2}+1
$$

and

$$
\pm C \xi=\int \frac{\mathrm{d} A}{\left(\frac{\chi A^{2}}{2}+1\right) \sqrt{P(I)}} .
$$

Using the explicit form of the calibrated invariance function $P(I)$, we will determine respectively the expressions of scalar potential $A(\xi)$ and of the component $g_{00}(\xi)$ of the metric tensor from the relations (26) and (25). Exploiting (19), we will express the other metric functions $g_{11}(\xi), g_{22}(\xi), g_{33}(\xi)$ and rewrite the analytical expression of the energy density $T_{0}^{0}(\xi)$. From the previous expressions, we will establish those of the energy density per unit invariant volume $T(\xi)$ and the total energy of the nonlinear induction interaction fields $E_{f}$ defined by the relations:

$$
\begin{aligned}
& T(\xi)=T_{0}^{0}(\xi) \sqrt{{ }_{-}^{3} g} \\
& E_{f}=\int_{0}^{\xi_{c}} T_{0}^{0} \sqrt{{ }_{-}^{3} g} \mathrm{~d} \xi .
\end{aligned}
$$

In generally from (11) one gets [18]:

$$
j^{v}=-\varphi_{, i} \varphi^{i} \psi_{I}(I) A^{v} .
$$

It allows to express the non-zero components of the 4-vector current density $j^{v}$ opposite:

$$
\begin{gathered}
j^{0}=-\mathrm{e}^{-2(\gamma+\alpha)} C^{2} P_{I}(I) A, \\
j^{1}=j^{2}=j^{3}=0 .
\end{gathered}
$$

The charge density $\rho_{e}(\xi)$, the charge density per unit invariant volume $\rho(\xi)$ and the total charge of elementary particles $Q$ verify the relations:

$$
\begin{gathered}
\rho_{e}(\xi)=-C^{2} \mathrm{e}^{-(2 \alpha+\gamma)} P_{I}(I) A, \\
\rho(\xi)=-C^{2} \mathrm{e}^{-2 \gamma} A P_{I}(I) \sin \theta,
\end{gathered}
$$

and

$$
Q=-C^{2} \int_{0}^{\xi_{c}} \mathrm{e}^{-2 \gamma} A \frac{\mathrm{d} P(I)}{\mathrm{d} I} \sin \theta \mathrm{d} \xi .
$$

In Section 3, we will determine the exact static spherical symmetric solutions to the Einstein equation, the electromagnetic and scalar nonlinear induction field equations using the calibrated invariance function:

$$
P(I)=P_{0}(N-\lambda I)^{2},
$$

where $P_{0}, N$ are dimensionless constants satisfying the conditions $N>\lambda I$ and $P(I)=1$ in the spatial infinity. 


\section{Exact Static Spherical Symmetric Solutions of the Einstein Equation, the Electromagnetic and Scalar Nonlinear Induction Field Equations}

Putting (35) into (26), the electric scalar potential is:

$$
A(\xi)=\sqrt{\frac{N}{\lambda\left(1-\sigma^{2}\right)}} \tanh (b \xi),
$$

where $b=C \sqrt{N P_{0} \lambda\left(1-\sigma^{2}\right)}, \quad \sigma^{2}=\frac{\chi N}{2 \lambda}$.

From (3), (19), (25) and (36), we establish the solutions of Einstein's equation:

$$
\begin{gathered}
g_{00}(\xi)=\frac{\cosh ^{2}(b \xi)-\sigma^{2}}{\left(1-\sigma^{2}\right) \cosh ^{2}(b \xi)}, \\
g_{11}(\xi)=-\frac{1-\sigma^{2}}{S^{4}} \frac{\cosh ^{2}(b \xi)}{\cosh ^{2}(b \xi)-\sigma^{2}}, \\
g_{22}(\xi)=\frac{g_{33}(\xi)}{\sin ^{2} \theta}=-\frac{1-\sigma^{2}}{S^{2}} \frac{\cosh ^{2}(b \xi)}{\cosh ^{2}(b \xi)-\sigma^{2}}
\end{gathered}
$$

where $S=S(k, \xi)$.

From (13), (19), (27), (28), (36), (37), (38) and (39), the chronometric invariant $I(\xi)$, the energy density $T_{0}^{0}(\xi)$, the energy density per unit invariant volume $T(\xi)$ and the total energy of nonlinear induction fields $E_{f}$ verify:

$$
\begin{gathered}
I(\xi)=\frac{N \sinh ^{2}(b \xi)}{\lambda\left[\cosh ^{2}(b \xi)-\sigma^{2}\right]}, \\
T_{0}^{0}(\xi)=\frac{S^{4} N^{2} P_{0} C^{2}}{2 \cosh ^{2}(b \xi)}\left[\frac{1-\sigma^{2}}{\cosh ^{2}(b \xi)-\sigma^{2}}+\frac{1}{\cosh ^{2}(b \xi)}-\frac{4 \sinh ^{2}(b \xi)}{\cosh ^{2}(b \xi)-\sigma^{2}}\right], \\
T(\xi)=R_{1}\left[\frac{\left(1-\sigma^{2}\right) \cosh (b \xi)}{\left[\cosh ^{2}(b \xi)-\sigma^{2}\right]^{5 / 2}}+\frac{\cosh ^{-1}(b \xi)}{\left[\cosh ^{2}(b \xi)-\sigma^{2}\right]^{5 / 2}}\right. \\
\left.-\frac{4 \sinh ^{2}(b \xi) \cosh (b \xi)}{\left[\cosh ^{2}(b \xi)-\sigma^{2}\right]^{5 / 2}}\right], \\
E_{f}=R_{1}\left[\left(1-\sigma^{2}\right) E_{f_{1}}+E_{f_{2}}-4 E_{f_{3}}\right],
\end{gathered}
$$

where

$$
\begin{gathered}
R_{1}=\frac{N^{2} P_{0} C^{2}}{2}\left(1-\sigma^{2}\right)^{3 / 2} \sin \theta, \\
E_{f_{1}}=\int_{0}^{\xi_{c}} \frac{\cosh (b \xi)}{\left[\cosh ^{2}(b \xi)-\sigma^{2}\right]^{5 / 2}} \mathrm{~d} \xi, \\
E_{f_{2}}=\int_{0}^{\xi_{c}} \frac{1}{\cosh (b \xi)\left[\cosh ^{2}(b \xi)-\sigma^{2}\right]^{3 / 2}} \mathrm{~d} \xi,
\end{gathered}
$$




$$
E_{f_{3}}=\int_{0}^{\xi_{c}} \frac{\sinh ^{2}(b \xi) \cosh (b \xi)}{\left[\cosh ^{2}(b \xi)-\sigma^{2}\right]^{5 / 2}} \mathrm{~d} \xi
$$

After a series of mathematical transformations, we obtain $E_{f_{1}}$ and $E_{f_{2}}$ :

$$
\begin{gathered}
E_{f_{1}}=\frac{R_{2} \sinh \left(b \xi_{c}\right)}{\left[\cosh ^{2}\left(b \xi_{c}\right)-\sigma^{2}\right]^{3 / 2}}+\frac{2 R_{3} \sinh \left(b \xi_{c}\right)}{\sqrt{\cosh ^{2}\left(b \xi_{c}\right)-\sigma^{2}}} ; \\
E_{f_{2}}=R_{4}\left[\frac{2 \sigma}{\sigma^{2}-1} \frac{\sinh \left(b \xi_{c}\right)}{\sqrt{\cosh ^{2}\left(b \xi_{c}\right)-\sigma^{2}}}+\ln \left|\frac{\sigma \sinh \left(b \xi_{c}\right)-\sqrt{\cosh ^{2}\left(b \xi_{c}\right)-\sigma^{2}}}{\sigma \sinh \left(b \xi_{c}\right)+\sqrt{\cosh ^{2}\left(b \xi_{c}\right)-\sigma^{2}}}\right|\right],
\end{gathered}
$$

where

$$
\begin{gathered}
R_{2}=\frac{1}{3 b\left(1-\sigma^{2}\right)}, \\
R_{3}=\frac{1}{3 b\left(1-\sigma^{2}\right)^{2}}, \\
R_{4}=-\frac{1}{2 b \sigma^{3}} .
\end{gathered}
$$

The relation $E_{f_{3}}$ being difficult to integrate, we will deduce its location on $\left[0, \xi_{c}\right]$ after its extension on $[0,+\infty]$. To do this, we will use the formula of the standard table of integrals [26]:

$$
\begin{aligned}
& \int_{0}^{\infty} \frac{\sinh ^{\mu-1} x \cosh ^{\nu-1} x}{\left(\cosh ^{2} x-\beta\right)^{\rho}} \mathrm{d} x \\
& =2 B\left(\frac{\mu}{2}, 1+\rho-\frac{\mu+v}{2}\right) \times F\left(\rho, 1+\rho-\frac{\mu+v}{2} ; 1+\rho-\frac{v}{2}, \beta\right) ;
\end{aligned}
$$

$\beta \notin(1, \infty), \operatorname{Re}(\mu)>0,2 \operatorname{Re}(1+\rho)>\operatorname{Re}(v+\mu)$. In this expression, $B(a, b)$ is the Bêta function and $F(a, b ; c, m)$ is the Gauss hyper-geometric function. So, we obtain:

$$
\begin{gathered}
E_{f_{3}}<\int_{0}^{\infty} \frac{\sinh ^{2}(b \xi) \cosh (b \xi)}{\left[\cosh ^{2}(b \xi)-\sigma^{2}\right]^{5 / 2}} \mathrm{~d} \xi, \\
E_{f_{3}}<F\left(\frac{5}{2} ; 1 ; \frac{5}{2} ; \sigma^{2}\right) \times 2 B\left(\frac{3}{2}, 1\right), \\
E_{f_{3}}<\frac{4}{3\left(1-\sigma^{2}\right)} .
\end{gathered}
$$

Thus,

$$
E_{f}<\infty \text {. }
$$

Also, the expressions (32), (33) and (34) are transformed into:

$$
\rho_{e}(\xi)=\frac{2 P_{0} C^{2} S^{4} N^{3 / 2} \sqrt{\lambda} \sinh (b \xi)}{\cosh ^{2}(b \xi) \sqrt{\cosh ^{2}(b \xi)-\sigma^{2}}},
$$




$$
\begin{gathered}
\rho(\xi)=2 C^{2} P_{0} \sqrt{\lambda}\left[N\left(1-\sigma^{2}\right)\right]^{3 / 2} \frac{\cosh (b \xi) \sinh (b \xi)}{\left[\cosh ^{2}(b \xi)-\sigma^{2}\right]^{2}} \sin \theta, \\
Q=-C N P_{0}^{1 / 2}\left(1-\sigma^{2}\right)\left[\frac{1}{\cosh ^{2}\left(b \xi_{c}\right)-\sigma^{2}}-\frac{1}{1-\sigma^{2}}\right] \sin \theta .
\end{gathered}
$$

In Section 4, we will discuss the influence of the function $S(k, \xi)$ on the obtained solutions.

\section{Discussion}

The electric scalar potential $A(\xi)$, the component $g_{00}(\xi)$ of the metric tensor, the energy and the charge densities per unit invariant volume $(T(\xi), \rho(\xi))$ are regular functions, independent of the concrete form of the function $S(k, \xi)$. Figure 1 below gives a graphical illustration:

In Figure 1(a), the solution of the equation to the electromagnetic and scalar fields of nonlinear induction and taking into account the own gravitational field of elementary particles describes a massless system contrary to the massive system obtained by [19].

In Figure 1(c), the energy density per unit invariant volume is an asymptotic and localized function. Its depth and width of localization depend on the value of the integration constants.

In Figure 1(d), the charge density per unit invariant volume is also an asymptotic and localized function, with depth and width of localization varying with the values of the constants integration.

Let us point out from (43) and (46) that, the total charge of elementary particles is a finite quantity and the total energy of fields is limited.

On the other hand, the components $g_{11}(\xi), g_{22}(\xi), g_{33}(\xi)$ of the metric tensor, the energy and charge densities $\left(T_{0}^{0}(\xi), \rho_{e}(\xi)\right)$ depend on the concrete form of the function $S(k, \xi)$.

In order to respect the regularity conditions [19] and [25], the obvious and trivial form $S(k, \xi)=\xi$ is often used. Let us analyze the solutions of the field and Einstein equations of Sect 0 , using all concrete forms of the function $S(k, \xi)$ because it also respects the boundary conditions.

\subsection{Case 1: $k>0$}

The concrete form of $S(k, \xi)$ is:

$$
S(k, \xi)=\frac{\sinh (k \xi)}{k} .
$$

The relations (47) (38), (39), (41) and (44) lead respectively to:

$$
\begin{gathered}
g_{11}(\xi)=-k^{4} \frac{1-\sigma^{2}}{\sinh ^{4}(k \xi)} \frac{\cosh ^{2}(b \xi)}{\cosh ^{2}(b \xi)-\sigma^{2}}, \\
g_{22}(\xi)=\frac{g_{33}(\xi)}{\sin ^{2} \theta}=-k^{2} \frac{1-\sigma^{2}}{\sinh ^{2}(k \xi)} \frac{\cosh ^{2}(b \xi)}{\cosh ^{2}(b \xi)-\sigma^{2}},
\end{gathered}
$$




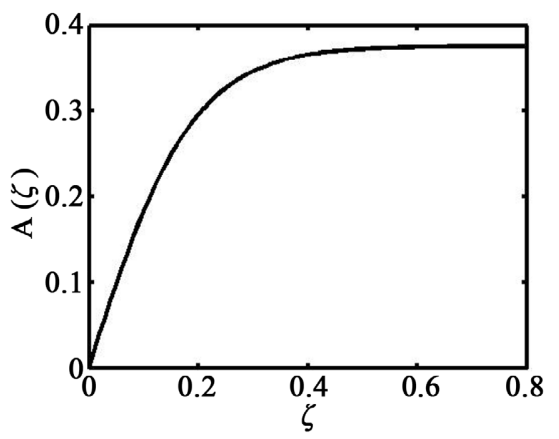

(a)

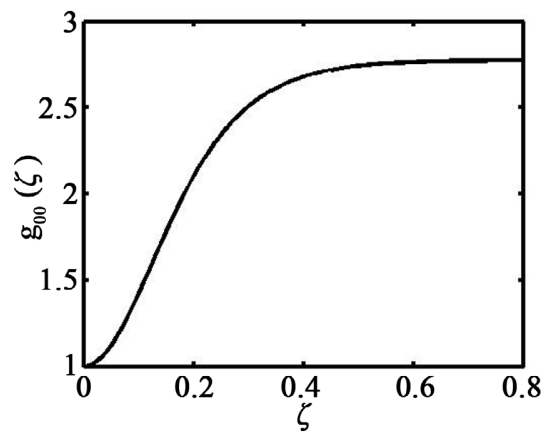

(b)

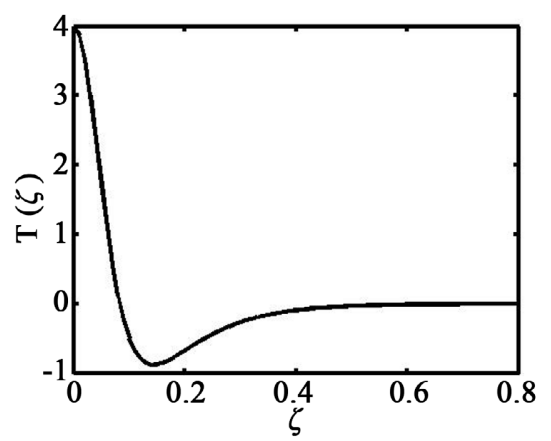

(c)

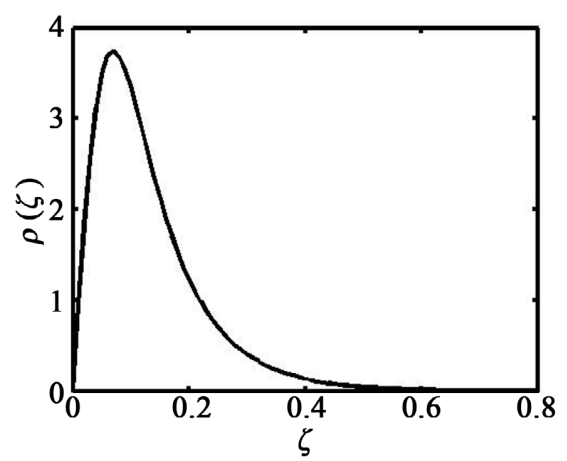

(d)

Figure 1. (a)-Electric scalar potential $A(\xi)$, (b)-Component $g_{00}(\xi)$ of the metric tensor, (c)-Energy density per unit invariant volume $T(\xi)$, (d)-Density of charge per unit invariant volume $\rho(\xi)$. The values of the parameters used for this simulation are: $\lambda=39 ; C=N=2 ; \chi=8 \pi$ and $\theta=\pi / 2$. 


$$
\begin{aligned}
T_{0}^{0}(\xi)= & \frac{N^{2} P_{0} C^{2} \sinh ^{4}(k \xi)}{2 k^{4} \cosh ^{2}(b \xi)}\left[\frac{1-\sigma^{2}}{\cosh ^{2}(b \xi)-\sigma^{2}}+\frac{1}{\cosh ^{2}(b \xi)}\right. \\
& \left.-\frac{4 \sinh ^{2}(b \xi)}{\cosh ^{2}(b \xi)-\sigma^{2}}\right], \\
\rho_{e}(\xi) & =\frac{2 P_{0} C^{2} N^{3 / 2} \sqrt{\lambda} \sinh ^{4}(k \xi)}{k^{4} \cosh ^{2}(b \xi)} \frac{\sinh (b \xi)}{\sqrt{\cosh ^{2}(b \xi)-\sigma^{2}}} .
\end{aligned}
$$

4.2. Case 2: $k<0$

$$
S(k, \xi)=\frac{\sin (k \xi)}{k} .
$$

The relation (52) in those (38), (39), (41) and (44) verify the equalities:

$$
\begin{gathered}
g_{11}(\xi)=-k^{4} \frac{1-\sigma^{2}}{\sin ^{4}(k \xi)} \frac{\cosh ^{2}(b \xi)}{\cosh ^{2}(b \xi)-\sigma^{2}}, \\
g_{22}(\xi)=\frac{g_{33}(\xi)}{\sin ^{2} \theta}=-k^{2} \frac{1-\sigma^{2}}{\sin ^{2}(k \xi)} \frac{\cosh ^{2}(b \xi)}{\cosh ^{2}(b \xi)-\sigma^{2}}, \\
T_{0}^{0}(\xi)=\frac{N^{2} P_{0} C^{2} \sin ^{2}(k \xi)}{2 k^{4} \cosh ^{2}(b \xi)}\left[\frac{1-\sigma^{2}}{\cosh ^{2}(b \xi)-\sigma^{2}}+\frac{1}{\cosh ^{2}(b \xi)}\right. \\
\left.-\frac{4 \sinh ^{2}(b \xi)}{\cosh ^{2}(b \xi)-\sigma^{2}}\right], \\
\rho_{e}(\xi)=\frac{2 P_{0} C^{2} N^{3 / 2} \sqrt{\lambda} \sin ^{4}(k \xi)}{k^{4} \cosh ^{2}(b \xi)} \frac{\sinh ^{2}(b \xi)}{\sqrt{\cosh ^{2}(b \xi)-\sigma^{2}}} .
\end{gathered}
$$

4.3. Case 3: $k=0$

$$
S(k, \xi)=\xi
$$

The relations (38), (39), (41), (44) give:

$$
\begin{gathered}
g_{11}(\xi)=-\frac{1-\sigma^{2}}{\xi^{4}} \frac{\cosh ^{2}(b \xi)}{\cosh ^{2}(b \xi)-\sigma^{2}}, \\
g_{22}(\xi)=\frac{g_{33}(\xi)}{\sin ^{2} \theta}=-\frac{1-\sigma^{2}}{\xi^{2}} \frac{\cosh ^{2}(b \xi)}{\cosh ^{2}(b \xi)-\sigma^{2}}, \\
T_{0}^{0}(\xi)=\frac{N^{2} P_{0} C^{2} \xi^{4}}{2 \cosh ^{2}(b \xi)}\left[\frac{1-\sigma^{2}}{\cosh ^{2}(b \xi)-\sigma^{2}}+\frac{1}{\cosh ^{2}(b \xi)}-\frac{4 \sinh ^{2}(b \xi)}{\cosh ^{2}(b \xi)-\sigma^{2}}\right], \\
\rho_{e}(\xi)=\frac{2 P_{0} C^{2} N^{3 / 2} \sqrt{\lambda} \xi^{4}}{\cosh ^{2}(b \xi)} \frac{\sinh (b \xi)}{\sqrt{\cosh ^{2}(b \xi)-\sigma^{2}}} .
\end{gathered}
$$

Figures 2-4 give a summary illustration of the properties of all solutions obtained in each of the Sections 4.1, 4.2 and 4.3.

In Figure 2(a), Figure 2(b), Figure 3(a), Figure 3(b), Figure 4(a) and Figure 4(b), all components of the metric tensor $g_{11}(\xi), g_{22}(\xi)$ and $g_{33}(\xi)$, exhibit 


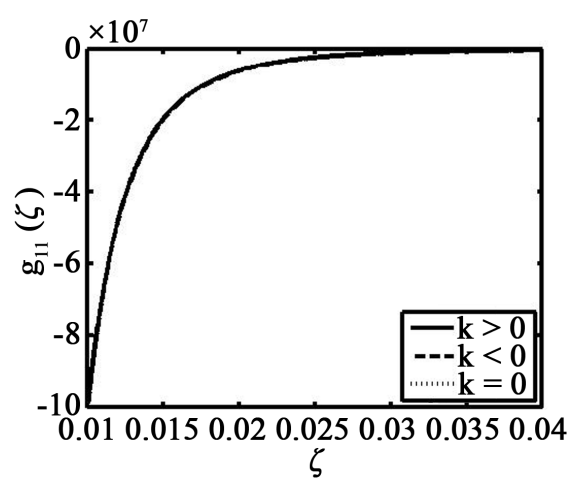

(a)

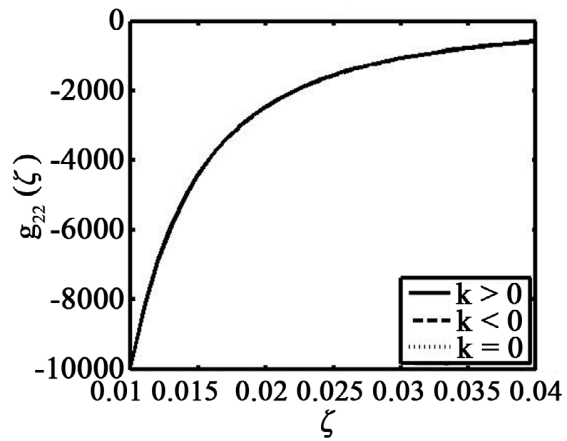

(b)

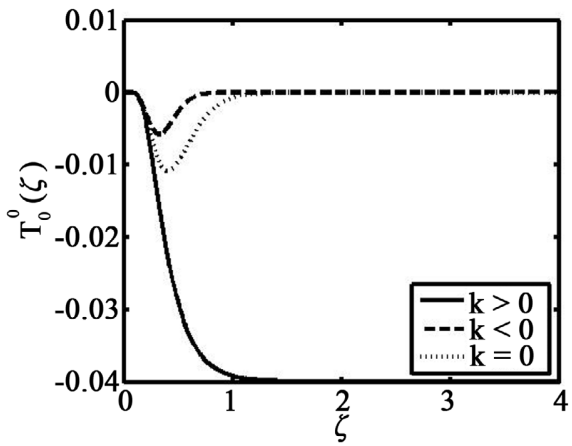

(c)

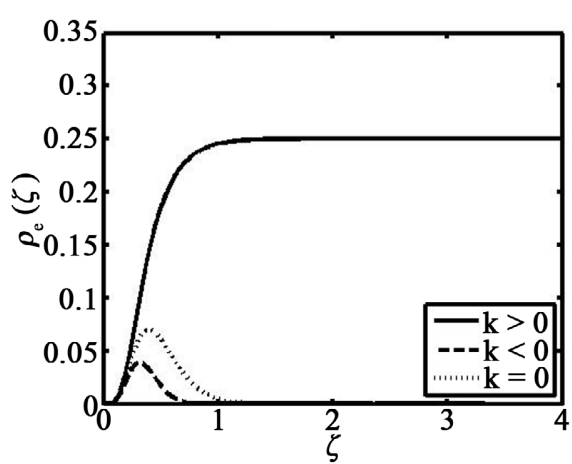

(d)

Figure 2. (a)-Component $g_{11}(\xi)$ of the metric tensor, (b)-Components $g_{22}(\xi)$ of the metric tensor, (c)-Energy densities $T_{0}^{0}(\xi),(\mathrm{d})$-Charge densities $\rho_{e}(\xi)$ using $k=0, \pm b / 2, \theta=\pi / 2$. 


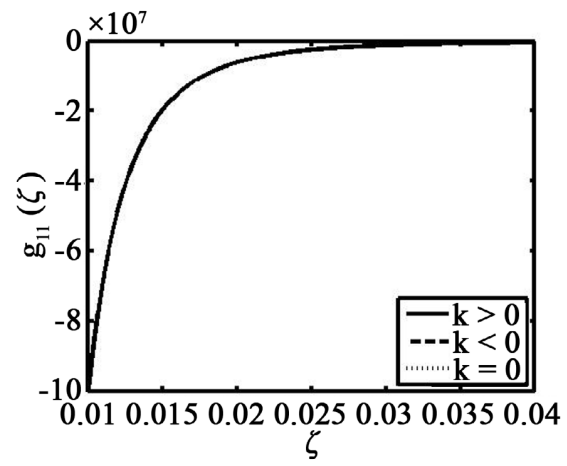

(a)

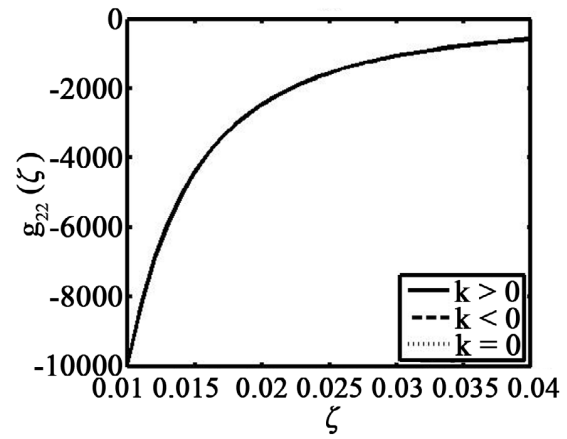

(b)

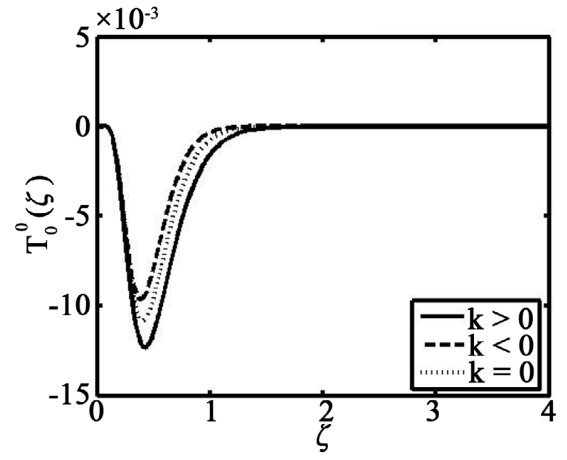

(c)

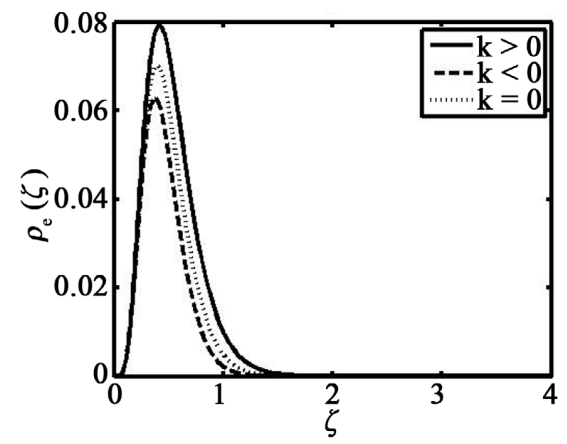

(d)

Figure 3. (a)-Component $g_{11}(\xi)$ of the metric tensor, (b)-Components $g_{22}(\xi)$ of the metric tensor, (c)-Energy densities $T_{0}^{0}(\xi),(\mathrm{d})$-Charge densities $\rho_{e}(\xi)$ using $k=0, \pm b / 5, \quad \theta=\pi / 2$. 


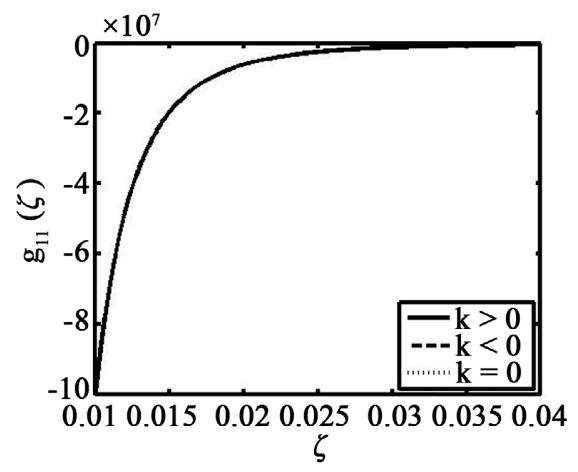

(a)

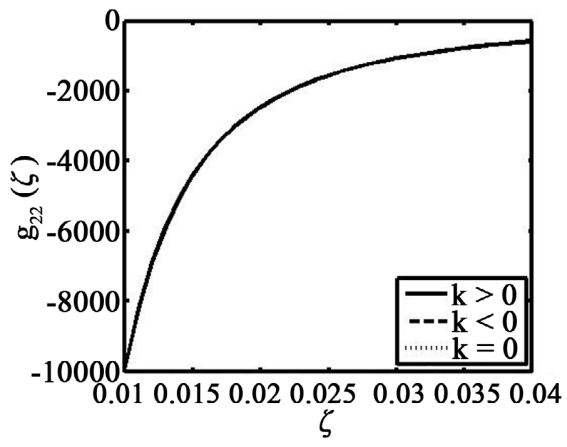

(b)

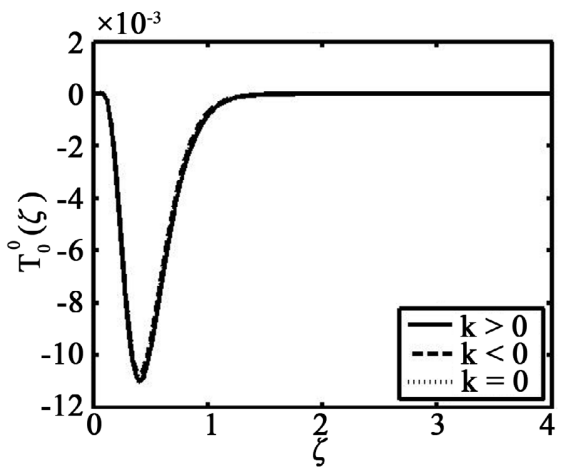

(c)

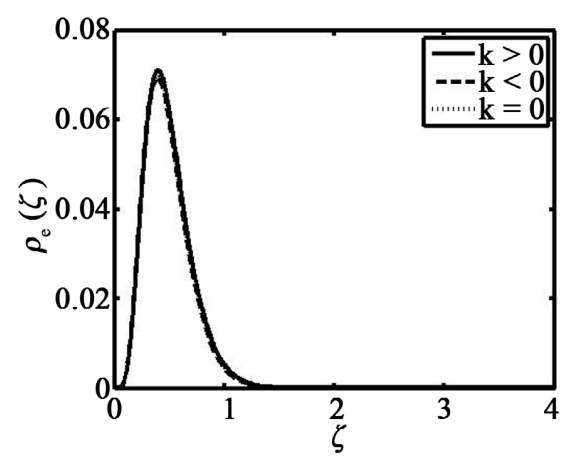

(d)

Figure 4. (a)-Component $g_{11}(\xi)$ of the metric tensor, (b)-Components $g_{22}(\xi)$ of the metric tensor, (c)-Energy densities $T_{0}^{0}(\xi)$, (d)-Charge densities $\rho_{e}(\xi)$ with $k=0, \pm b / 15$, $\theta=\pi / 2$. 
a gravitational singularity $\left(\mathrm{e}^{\beta}=\infty\right)$ that is uncontrollable by change of variable in infinite space whatever the form of the function $S(k, \xi)$ as stated in [27].

In Figure 2(c), Figure 3(c) and Figure 4(c), the energy densities $T_{0}^{0}(\xi)$ are asymptotic, localized functions, all canceling in infinite space. The width of the localization and the depth vary with the values of the integration constants, in particular that of $k$.

In Figure 2(d), Figure 3(d) and Figure 4(d), the charge densities $\rho_{e}(\xi)$ have the same properties as the energy densities of Figures 2 (c)-4(c) but are positive definite.

We will focus in Section 5 on the role of the own gravitational field of elementary particles on the soliton-like solutions.

\section{Solution in Flat Space-Time}

In the absence of the own gravitational field of elementary particles, the metric (2) becomes:

$$
\mathrm{d} s^{2}=\mathrm{d} t^{2}-\mathrm{d} \xi^{2}-\left[\mathrm{d} \theta^{2}+\sin ^{2}(\theta) \mathrm{d} \varphi^{2}\right] .
$$

The electromagnetic field Equation (17) takes the form:

$$
A^{\prime \prime}-C^{2} P_{I}(I) A=0 \text {, }
$$

which has the solution:

$$
\pm C \xi=\int \frac{\mathrm{d} A}{\sqrt{P(I)}} .
$$

Using (35), the relation (63) gives the expression of electric scalar potential:

$$
A(\xi)=\sqrt{\frac{N}{\lambda}} \tanh (u \xi)
$$

where $u=C \sqrt{N P_{0} \lambda}$.

The energy and charge densities per unit invariant volume $(T(\xi), \rho(\xi))$ verify the following expressions:

$$
\begin{gathered}
T_{0}^{0}(\xi)=\frac{T(\xi)}{\sin \theta}=\frac{P_{0} N^{2} C^{2}}{\cosh ^{4}(u \xi)}\left[1-2 \sinh ^{2}(u \xi)\right], \\
\rho(\xi)=\rho_{e}(\xi) \sqrt{{ }_{-}^{3} g}=2 C^{2} P_{0} N^{3 / 2} \sqrt{\lambda} \frac{\sinh (u \xi)}{\cosh ^{3}(u \xi)} \sin \theta .
\end{gathered}
$$

Figure 5 below, shows the role of the own gravitational field of elementary particles on the solutions established in Section 4.

In Figure 5(a), we see that in the absence of the own gravitational field of the elementary particles, the electric scalar potential (64) remains a regular but of smaller amplitude than the one obtained in Figure 1(a).

In Figure 5(b), the energy density per unit invariant volume has the same properties as those obtained by taking into account the own gravitational field of the elementary particles but with almost equal depth. 


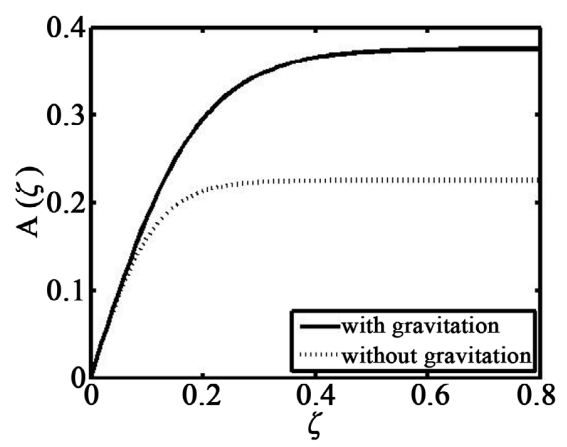

(a)

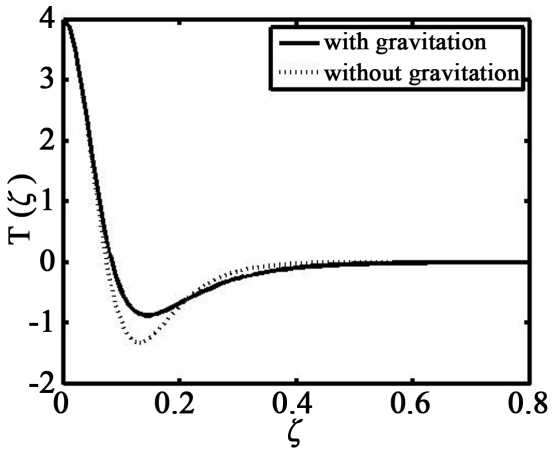

(b)

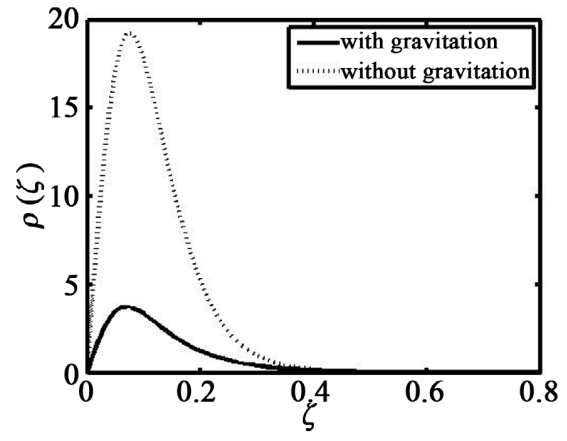

(c)

Figure 5. (a)-Electric scalar potential $A(\xi)$, (b)-Density of energy per unit invariant volume $T(\xi)$ and (c)-Density of charge per unit invariant volume $\rho(\xi)$.

In Figure 5(c), the charge density per unit invariant volume has a more extensive depth in the absence of the elementary particle gravitational field, is asymptotic and localized in an interval whose width depends on the integration constants.

The total energy of fields $E_{f}$ and the total charge $Q$ of elementary particles are given by the expressions:

$$
\begin{gathered}
E_{f}=\frac{C N^{3 / 2} P_{0}^{1 / 2}}{\lambda^{1 / 2}}\left[\tanh \left(u \xi_{c}\right)-\tanh ^{3}\left(u \xi_{c}\right)\right] \sin \theta, \\
Q=-\frac{2 C N P_{0}^{1 / 2}}{3}\left[\frac{1}{\cosh ^{3}\left(u \xi_{c}\right)}-1\right] \sin \theta .
\end{gathered}
$$




\section{Conclusion}

It has been proved that with all forms of the function $S(k, \xi)$, the electromagnetic and scalar nonlinear induction field equations, taking into account the own gravitational field of the elementary particles, are solvable. The obtained results show that, the component $g_{00}(\xi)$ of the metric tensor is a regular function. However, the other metric functions present a gravitational singularity in infinite space. All energy densities are localized and have a localization depth and width whose interval varies according to the values of the integration constants. The total energy of the nonlinear induction fields and the total charge of elementary particles are finite. These solutions are soliton-like and constitute a model able to describe the complex internal configuration of the elementary particles. In the near future, we will discuss the stability as well as the observability of the localized configurations obtained.

\section{Conflicts of Interest}

The authors declare no conflicts of interest regarding the publication of this paper.

\section{References}

[1] Bay, A. (n.d.) Particules élémentaires, Laboratoire de physique des hautes énergies EPFL, 2-4, 342. http://lphe.epfl.ch/bay

[2] Rajaraman, R. (1982) An Introduction to Solitons and Instantons in Quantum Field Theory. North-Holland Publishing Company, New York

[3] Bogoliubov, N.N. and Shirkov, D.V. (1976) An Introduction to the Theory of Quantized Fields. Nauka, Moscow.

[4] Perring, J.K. and Skyrme, T.H.R. (1962) A Model Unified Field Equation. Nuclear Physics, 31, 550-555. https://doi.org/10.1016/0029-5582(62)90774-5

[5] Rybakov, Yu. (1985) Particles Structure in Nonlinear Field Theory. Peoples' Friendship University of Russia, Moscow.

[6] Dodd, R.K., Eilbeck, J.C., Gibbon, J.D. and Morris, H.C. (1982) Solitons and Nonlinear Wave Equations. Academic Press, London.

[7] Scott, A.C., Chu, F.Y.F. and McLaughlin, D.W. (1973) The Soliton: A New Concept in Applied Science. Proceedings of the IEEE, 61, 1443-1483.

https://doi.org/10.1109/PROC.1973.9296

[8] Shikin, G.N. (1991) Basics of Soliton Theory in General Relativity. URSS Publishers, Moscow.

[9] Shikin, G.N. (1995) Theory of Solitons in General Relativity. URSS (Undergraduate Research Support Scheme), Moscow.

[10] Korteweg, D.J. and De Vries, G. (1895) On the Change of Form of Long Waves Advancing in a Rectangular Canal and on a New Type of Long Stationary Waves. The London, Edinburgh, and Dublin Philosophical Magazine and Journal of Science, 36, 422-443. https://doi.org/10.1080/14786449508620739

[11] Russell, J.S. (1844) Report on Waves. Report of the 14th Meeting of British Association for the Advancement of Science, York, September 1844, 311-390.

[12] Kerr, R. (1963) Gravitational Field of a Spinning Mass as an Example of Algebrai- 
cally Special Metrics. Physical Review Letters, 11, 237-238. https://doi.org/10.1103/PhysRevLett.11.237

[13] Wiltshire, D., Visser, M. and Scott, S. (2009) The Kerr Space-Time: Rotating Black Holes in General Relativity. Cambridge University Press, Cambridge.

[14] Wald, R.M. (1984) General Relativity. Chicago University Press, Chicago. https://doi.org/10.7208/chicago/9780226870373.001.0001

[15] Pellicer, R. and Torrence, R.J. (1969) Nonlinear Electrodynamics and General Relativity. Journal of Mathematical Physics, 10, 1718-1723.

https://doi.org/10.1063/1.1665019

[16] Plebanski, J. (1966) Non-Linear Electrodynamics-A Study. C.I.E.A. del I.P.N., Mexico City.

[17] Bronnikov, K.A., Melnikov, V.N., Shikin, G.N. and Staniukovich, K.P. (1979) Scalar, Electromagnetic, and Gravitational Fields Interaction: Particlelike Solutions. Annals of physics, 118, 84-107. https://doi.org/10.1016/0003-4916(79)90235-5

[18] Kulyabov, D.S., Rybakov, Yu.P., Shikin, G.N. and Yuschenko, L.P. (1999) Kink-Like Configurations of Interacting Scalar, Electromagnetic, and Gravitational Fields. Bulletin of Peoples Friendship University of Russia, Series Physics, No. 1, 56-60. http://arxiv.org/abs/math-ph/9902011v1

[19] Rybakov, Yu.P., Shikin, G.N. and Saha, B. (1997) Solitons of Nonlinear Scalar Electrodynamics in General Relativity. International Journal of Theoretical Physics, 36, 1475-1494. https://doi.org/10.1007/BF02435941

[20] Adomou, A., Edou, J. and Massou S. (2019) Soliton-Like Spherical Symmetric Solution of the Nonlinear Spinor Field Equation Depending on the Invariant Function $I_{p}$ $=P^{2}$ in the General Relativity. Journal of Applied Mathematics and Physics, 7, 2818-2835. https://doi.org/10.4236/jamp.2019.711194

[21] Adomou, A., Edou, J. and Massou, S. (2019) Soliton-Like Spherical Symmetric Solutions of the Nonlinear Spinor Field equations in General Relativity. International Journal of Applied Mathematics and Theoretical Physics, 5, 118-128.

[22] Adanhoumè, A., Adomou, A. and Hounkonnou, M.N. (2012) Nonlinear Spinor Field Equations in Gravitational Theory: Spherical Symmetric Soliton-Like Solutions. Journal of Modern Physics, 3, 935-942. http://arxiv.org/abs/1211.3388v1 https://doi.org/10.4236/jmp.2012.39122

[23] Adomou, A., Edou, J., Hontonfinde, V.I.S. and Massou, S. (2020) Exact Soliton-Like Spherical Symmetric Solutions of the Heisenberg-Ivanenko Type Nonlinear Spinor Field Equation in Gravitational Theory. Journal of Applied Mathematics and Physics, 8, 1236-1254. https://doi.org/10.4236/jamp.2020.87094

[24] Bronnikov, K.A. and Rubin, S.G. (2012) Black Holes, Cosmology and Extra Dimensions. World Scientific Publishing Company, Singapore.

https://doi.org/10.1142/8302

[25] Rybakov, Yu.P., Shikin, G.N. and Saha, B. (1998) Droplets in General Relativity: Exact Self-Consistent Solutions to the Interacting Scalar and Electromagnetic Field Equations. Gravitation and Cosmology, 4, 114-120. https://arxiv.org/abs/gr-qc/9603032v1

[26] Gradshteyn, I.S. and Ryzhik, I.M. (2007) Table of Integrals, Series, and Products. Academic Press, Cambridge.

[27] Vasset, N. (2009) Quelques aspects des horizons de trous noirs en relativité numérique. Thèse de l'Université de Paris VII . 